\title{
TAMING FREE CIRCLE ACTIONS
}

\author{
ALLAN L. EDMONDS ${ }^{1}$
}

\begin{abstract}
It is shown that an arbitrary free action of the circle group on a closed manifold of dimension at least six is concordant to a "tame" action (so that the orbit space is a manifold). A consequence is that the concordance classification of arbitrary free actions of the circle on a simply connected manifold is the same as the equivariant homeomorphism classification of free tame actions.
\end{abstract}

Consider a free action of a compact Lie group $G$ of positive dimension on a topological manifold $M$. Such an action is called tame if the orbit space $M / G$ is a manifold and wild otherwise. Starting with a tame free action on $M$ one may obtain uncountably many inequivalent wild actions on $M$ by collapsing out noncellular arcs in $M / G$, provided $M / G$ has dimension at least three. For an exposition of this construction see L. Lininger [9].

The existence of such wild actions makes classification theorems difficult without a tameness hypothesis. In this paper a standard concordance equivalence relation on the set of free actions on a manifold is considered. At least for the circle group $S^{1}$, the concordance equivalence relation makes tame classification theorems applicable in the general case.

Define a $G$-concordance to be a $G$-action on $M \times[0,1]$ preserving $M$ $\times 0$ and $M \times 1$. Actions $\varphi$ and $\psi$ of $G$ on $M$ are $G$-concordant if there is a $G$ concordance $\theta$ on $M \times[0,1]$ such that $\theta \mid M \times 0=\varphi$ and $\theta \mid M \times 1=\psi$.

MAIN THEOREM. If $M$ is a closed topological $n$-manifold, $n \geqslant 6$, then any free $S^{1}$-action on $M$ is $S^{1}$-concordant to a tame action.

There are both a relative version and a suitable uniqueness statement. One might conjecture a similar result for arbitrary compact Lie groups.

C.T.C. Wall [12] has classified equivariant homeomorphism classes of tame free $S^{1}$-actions on spheres $S^{2 n+1}, n \geqslant 3$, by classifying the possible orbit spaces using the theory of surgery. Wall conjectured [12, p. 192] that even in the wild case this calculation can be interpreted to give a correct result. The Main Theorem provides an affirmative answer to this conjecture. See Corollary 3.3.

Received by the editors April 12, 1976.

AMS (MOS) subject classifications (1970). Primary 57E10, 57A99.

Key words and phrases. Circle group, wild action, tame action, concordance, classification of group actions on manifolds.

${ }^{1}$ Supported in part by NSF Grant GP 33960X. 
Ingredients in the proof of the Main Theorem are the result of J. West [13] that a compact absolute neighborhood retract (ANR) has the homotopy type of a finite $\mathrm{CW}$ complex, the consequent extension of simple homotopy theory to compact ANR's by T. Chapman [4], and the results of L. Siebenmann's thesis [10] in the form of the "topological ribbon theorem" [11]. (Actually the references to work of West and Chapman can be replaced by citing R. Kirby and Siebenmann [8], at a cost of complicating some of the preliminary material.)

In $\$ 1$ some preliminary material is collected, including a precise statement of the Ribbon Theorem; the Main Theorem is proved in \$2; and several applications are given in $\$ 3$.

1. Preliminary material. All spaces considered here will be separable metric. An $n$-manifold is a space $M$ locally homeomorphic to half-space $\left\{\left(x_{1}, x_{2}, \ldots\right.\right.$, $\left.\left.x_{n}\right): x_{n} \geqslant 0\right\}$ in euclidean $n$-space $\mathbf{R}^{n}$. The boundary of $M$ is denoted $\partial M$.

The symbols $\cong, \approx$, and $\simeq$ denote, respectively, homeomorphism, isomorphism, and homotopy equivalence.

We shall assume the basic facts about (separable metric) absolute neighborhood retracts (ANR's). A convenient reference is K. Borsuk [2].

Using the theory of Hilbert cube manifolds J. West [13] has shown that every compact ANR has the homotopy type of a finite $\mathrm{CW}$ complex. $\mathrm{T}$. Chapman [4] has further shown that the ordinary simple homotopy theory of finite CW complexes carries over full-blown to the setting of compact ANR's. Recall that a surjective map $f: X \rightarrow Y$ of ANR's is cell-like (CE) if (among several equivalent definitions) for each $y \in Y$ and neighborhood $U$ of $f^{-1}(y)$ in $X$ there is a neighborhood $V$ of $f^{-1}(y)$ in $U$ such that the inclusion map $V \rightarrow U$ is null-homotopic. A homotopy equivalence $h: X \rightarrow Y$ of compact ANR's is defined to be simple if there are a compact ANR $Z$ and CE maps $f: Z \rightarrow X$ and $g: Z \rightarrow Y$ such that $h f \simeq g$. In general there is defined in the Whitehead group $\mathrm{Wh}\left(\pi_{1} X\right)$ a torsion invariant $\tau(h)$ which vanishes if and only if the homotopy equivalence $h$ is simple. Chapman has shown that these definitions generalize the usual definitions for $\mathrm{CW}$ complexes and cellular maps and that all the standard properties of Whitehead torsion (see, e.g., [6]) hold in this setting.

The following result generalizes $[1,(2.1)]$ and will be needed in $\S 2$.

LEMMA 1.1. Let $p: E \rightarrow B$ be a locally trivial fiber bundle with base $B$ and fiber $F$ compact ANR's and let $h: A \rightarrow B$ be a simple homotopy equivalence of compact $A N R$ 's. Then the natural map $h^{*} E \rightarrow E$ is also a simple homotopy equivalence, where $h^{*} E=\{(a, e) \in A \times E: h(a)=p(e)\}$ is the total space of the induced bundle over $A$.

Proof. One easily verifies that $E$ and $h^{*} E$ are compact ANR's using the local characterization of such spaces (see, e.g., [2, p. 97]). The ladder connecting the exact homotopy sequences of the bundles $h^{*} E$ and $E$ shows that $h^{*} E \rightarrow E$ is a homotopy equivalence. By definition there are a compact 
ANR $Z$ and CE maps $f: Z \rightarrow A$ and $g: Z \rightarrow B$ such that $h f \simeq g$. The natural maps of induced bundles $f^{*} h^{*} E \rightarrow h^{*} E$ and $g^{*} E \rightarrow E$ are easily seen to be CE using properties of pullback diagrams. But there is an isomorphism between $g^{*} E$ and $f^{*} h^{*} E$ obtained using a homotopy between $h f$ and $g$. Hence there are CE maps $g^{*} E \rightarrow h^{*} E$ and $g^{*} E \rightarrow E$ of the sort needed to show that $h^{*} E \rightarrow E$ is a simple homotopy equivalence.

The above description of simple homotopy types necessarily coincides with that given earlier by R. Kirby and L. Siebenmann [8] for topological [TOP] manifolds. In the case of TOP manifolds we shall need the s-cobordism theorem which is proved using TOP handlebody theory. We shall also need the following "topological ribbon theorem," which is also proved by following the outline of the proof of the corresponding result in the smooth category using TOP handlebody theory.

A ribbon $W$ is a noncompact, connected $n$-manifold such that (i) $\partial W$ $\cong N \times \mathbf{R}$ for some compact $(n-2)$-manifold $N$ and (ii) there exist a finite complex $K$ and a proper (the inverse image of any compactum is compact) map $r: K \times \mathbf{R} \rightarrow W$ inducing an isomorphism $\pi_{1} K \approx \pi_{1} W$ such that $r$ is a retraction in the category of proper homotopy classes of proper maps.

Any ribbon $W$ has two ends; call one $\varepsilon$. There is an invariant $\sigma(\varepsilon)$ in the reduced projective class group $\tilde{K}_{0}\left(\mathbf{Z}\left[\pi_{1} W\right]\right)$; up to sign it is the obstruction to a suitably small closed submanifold neighborhood $V$ of $\varepsilon$ in $W$ having the homotopy type of a finite complex.

RibBon TheOREM [10], [11]. A ribbon $W$ of dimension at least 6 is homeomorphic to $M \times \mathbf{R}$ for some compact manifold $M$ if and only if the invariant $\sigma(\varepsilon)=0$. If $\sigma(\varepsilon)=0$ the asserted product structure can be chosen to extend a given product structure on $\partial W$.

2. Proof of the Main Theorem. Following two lemmas the Main Theorem is proved in the needed relative version as Theorem 2.3.

Lemma 2.1. Let $S^{1}$ act freely on an n-manifold $M$. Then $M / S^{1} \times \mathbf{R}$ is a manifold with $\partial\left(M / S^{1} \times \mathbf{R}\right)=(\partial M) / S^{1} \times \mathbf{R}$.

Proof. By a theorem due originally to A. Gleason, or its generalization, the Slice Theorem [3, II.5.4], the orbit map $p: M \rightarrow M / S^{1}$ is a locally trivial fiber bundle with fiber $S^{1}$. Therefore each point of $M / S^{1}$ is contained in an open set $U$ such that $p^{-1}(U) \cong U \times S^{1}$. Since $M$ is an $n$-manifold, the open set $p^{-1}(U)$ is an $n$-manifold with boundary

$$
\partial p^{-1}(U)=p^{-1}(U) \cap \partial M=p^{-1}(U \cap p(\partial M)) .
$$

Thus the covering space $U \times \mathbf{R}$ of $U \times S^{1}$ is a manifold with $\partial(U \times \mathbf{R})$ $=\left(U \cap \partial M / S^{1}\right) \times \mathbf{R}$. Since $M / S^{1} \times \mathbf{R}$ is covered by these open sets $U \times \mathbf{R}$, the lemma follows.

REMARK. If $G$ is any compact Lie group acting freely on $M$ then a similar argument shows that $M / G \times \mathbf{R}^{d}$ is a manifold, where $d$ is the dimension of $G$. 
If it could be shown that, in fact, $M / G \times \mathbf{R}$ is a manifold, then the main result of this paper would also hold for general compact Lie groups $G$.

LEMMA 2.2. Let $S^{1}$ act freely on a topological n-manifold $M$. Then $M / S^{1}$ is an ANR.

Proof. Since $M / S^{1} \times \mathbf{R}$ is a manifold it is an ANR. Thus $M / S^{1}$ is an ANR being a retract of one.

REMARK. This is actually a very special case of a general theorem of P. E. Conner and E. E. Floyd [7].

A $G$-concordance on $M \times I, I=[0,1]$, is said to be relative to $X \subset M$ if the action restricted to $X \times I$ has the form $g(x, t)=(\operatorname{pr} g(x, 0), t)$, where $\mathrm{pr}$ denotes projection on the first factor.

THEOREM 2.3. Let $S^{1}$ act freely on the compact n-manifold $M$ where $n$ $\geqslant 7$ or $n \geqslant 6$ and the action restricted to $\partial M$ is tame. Then the action is $S^{1}$ concordant to a tame action and such an $S^{1}$-concordance can be chosen to be relative to $\partial M$ if the given action is tame on $\partial M$.

Proof. First consider the special case when the action on $\partial M$ is tame. Let $W=M / S^{1} \times \mathbf{R}$. Now by (2.2) $M / S^{1}$ is a compact ANR, so by West's theorem [13] there is a finite CW complex $K$ and a homotopy equivalence $f: K$ $\rightarrow M / S^{1}$. By (2.1) $W$ is a manifold with $\partial W=\partial M / S^{1} \times \mathbf{R}$. Define $r: K \times \mathbf{R}$ $\rightarrow W$ by $r=f \times 1$. Then clearly $r$ is a proper homotopy equivalence (not just a proper retraction), so that $W$ is a ribbon. Let $\varepsilon$ denote the "positive" end of $W$. In fact the invariant $\sigma(\varepsilon)=0$. To see this let $U \subset M / S^{1} \times(0, \infty)$ be a closed submanifold neighborhood of $\varepsilon$. For large $t>0, M / S^{1} \times t \subset$ int $U$. Then the ANR $U$ strong deformation retracts onto the compactum $C$ $=U-M / S^{1} \times(t, \infty)$. Thus $C$ is a compact ANR; by [13] $C$ has the homotopy type of a finite complex; hence $U$ also has the homotopy type of a finite complex; and so $\sigma(\varepsilon)=0$.

Therefore by the Ribbon Theorem there is a manifold $N$ with $\partial N=\partial M / S^{1}$ such that the identity $\partial M / S^{1} \times \mathbf{R} \rightarrow \partial M / S^{1} \times \mathbf{R}$ extends to a homeomorphism $N \times \mathbf{R} \rightarrow M / S^{1} \times \mathbf{R}$. Identify $N \times \mathbf{R}$ and $M / S^{1} \times \mathbf{R}$ via this homeomorphism.

For sufficiently large $t>0, N \times t$ and $M / S^{1} \times 0$ are disjoint and together separate $W$ into three regions one of which, call it $V$, is compact. By shrinking $M / S^{1} \times(-\infty, 0]$ to $M / S^{1} \times 0$ and $N \times[t, \infty)$ to $N \times t$, it is seen that $V$ is a strong deformation retract of $W$ and so is an ANR. Moreover, the inclusions $M / S^{1} \times 0 \rightarrow V$ and $N \times t \rightarrow V$ are homotopy equivalences.

According to Chapman [4] there is then a Whitehead torsion

$$
\tau_{0}=\tau(V, N \times t)
$$

defined in $\operatorname{Wh}(\pi), \pi=\pi_{1} W$. By altering the choice of $N$ as follows we may assume $\tau_{0}=0$. By the existence theorem for Whitehead torsions of $h$ cobordisms, there is a compact submanifold $N^{\prime}$ of $N \times(t, 3 t)$ such that 
$\partial N^{\prime}=\partial N \times 2 t$, and the region $V^{\prime}$ between $N \times t$ and $N^{\prime}$ is an $h$-cobordism with torsion $\tau\left(V^{\prime}, N^{\prime}\right)=-\tau_{0}$ in $\mathrm{Wh}(\pi)$. Since $N$ and $N^{\prime}$ are $h$-cobordant, $N \times \mathbf{R} \cong N^{\prime} \times \mathbf{R}$ (and this product structure can be prescribed on the boundary). By the Sum Theorem for Whitehead torsion, $\tau\left(V \cup V^{\prime}, N^{\prime}\right)=0$. Thus we may assume that $N$ was originally chosen so that $\tau(V, N)=0$.

Now $M / S^{1} \times \mathbf{R}$ is the orbit space for an obvious $S^{1}$-action on $M \times \mathbf{R}$. Let $V^{\sim}$ be the region of $M \times \mathbf{R}$ over $V$ and $N^{\sim}$ be the portion of $V^{\sim}$ over $N \times t$. Then $V^{\sim}$ is a manifold, for $V^{\sim}-M \times 0$ is the total space of an $S^{1}$-bundle over the manifold $V-M / S^{1} \times 0$ while $M \times 0$ has a collar neighborhood $M \times[0, \delta)$ in $V^{\sim}$. Moreover $\partial V^{\sim}=M \times 0 \cup \partial M \times[0, t] \cup N^{\sim}$.

Now by (1.1) the inclusions $M \rightarrow V^{\sim}$ and $N^{\sim} \rightarrow V^{\sim}$ are simple homotopy equivalences since $M / S^{1} \times 0 \rightarrow V$ and $N \times t \rightarrow V$ are. Thus the $s$-cobordism theorem implies that $V^{\sim} \cong M \times I$, with prescribed product structure for $\partial M \times I$, and so $V^{\sim}$ provides the desired relative $S^{1}$-concordance to the tame action on $N^{\sim}$.

It remains to consider the case when $M$ has boundary on which the given action is not already tame. According to the first case considered, there is an $S^{1}$-concordance on $\partial M \times I$ between the given action on $\partial M \times 0$ and a tame action on $\partial M \times 1$. Using a boundary collar in $M$ this $S^{1}$-concordance can be extended to an $S^{1}$-concordance on $M \times I$, represented schematically in Figure 1 .

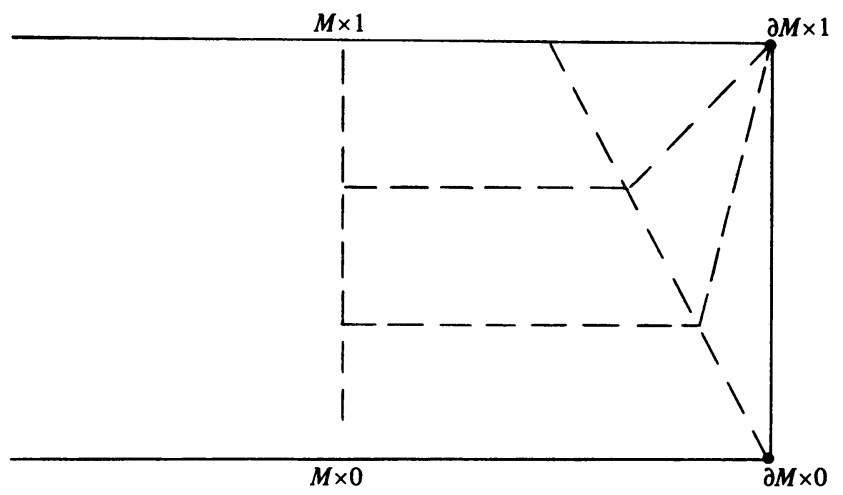

FIGURE 1

Further details of the construction of this $S^{1}$-concordance extension are left to the reader.

Thus the given action is concordant to an action which is tame on the boundary. The first case now applies to the latter action to complete the proof.

Corollary 2.4 (UniQueness). If $M$ is a closed $n$-manifold, $n \geqslant 5$, then $S^{1}$ concordant tame free $S^{1}$-actions on $M$ are tamely $S^{1}$-concordant. 
Proof. Apply the relative version of $(2.3)$ to a possibly wild $S^{1}$-concordance on $M \times I$, tame on the boundary $M \times\{0,1\}$. One only has to check that any concordance between free actions is itself necessarily a free action. This follows immediately from the relative form of the Smith theorems for $\mathbf{Z}_{p}$ actions on $(M \times I, M \times 0)$. See for example, [3, pp. 144f].

3. Applications. The following is an immediate consequence of (2.3) and the construction of [9].

COROLlaRY 3.1. If a compact $n$-manifold $M, n \geqslant 7($ or $n \geqslant 6$ if $\partial M=\varnothing)$ admits a free $S^{1}$-action, then it admits a tame $S^{1}$-action and uncountably many inequivalent wild actions.

Corollary 3.2. A compact $n$-manifold $M, n \geqslant 7$ (or $n \geqslant 6$ if $\partial M=\varnothing)$ admits at most countably many $S^{1}$-concordance classes of free $S^{1}$-actions.

Proof. According to (2.3) and (2.4) it suffices to observe that the compact $n$-manifold $M$ admits at most countably many inequivalent tame free $S^{1}$ actions. The orbit spaces are then manifolds and according to [5] there are only countably many possible orbit spaces up to homeomorphism. Now the principal $S^{1}$-bundles over a given compact $(n-1)$-manifold $N$ are in one-toone correspondence with the countable set

$$
\left[N ; B S O_{2}\right] \approx\left[N ; \mathbf{C P} P^{\infty}\right] \approx H^{2}(N ; \mathbf{Z})
$$

Only countably many of these $S^{1}$-bundles have total space $M$.

REMARK. One might conjecture that a version (3.2) holds for arbitrary compact Lie groups $G$, perhaps even for $G$-concordance classes of arbitrary $G$ actions.

The following corollary verifies the conjecture of C.T.C. Wall mentioned in the introduction.

COROLlaRy 3.3. For $n \geqslant 3$ the set of $S^{1}$-concordance classes of free $S^{1}$-actions on $S^{2 n+1}$ is in one-to-one correspondence with the set of equivariant homeomorphism classes of tame free $S^{1}$-actions on $S^{2 n+1}$.

Proof. By (2.3) and (2.4) each free $S^{1}$-action is $S^{1}$-concordant to a tame action and $S^{1}$-concordant tame actions are tamely concordant. Thus it suffices to show that tamely concordant tame free $S^{1}$-actions are equivalent. But the orbit space of such a tame concordance would be an $h$-cobordism of 1 connected manifolds and hence be a product $N \times I$ by the $h$-cobordism theorem. Lifting this product structure to the total space of the $S^{1}$-bundle over $N \times I$ shows the two actions are equivalent.

REMARK. In general it might be worthwhile to strengthen the definition of a free $G$-concordance on $M \times I$ to include the requirement that the inclusions $M \times 0 / G \rightarrow M \times I / G$ and $M \times 1 / G \rightarrow M \times I / G$ be simple homotopy equivalences, so that, as in (3.3), a tame free $G$-concordance is always a product. 


\section{REFERENCES}

1. D. R. Anderson, The Whitehead torsion of the total space of a fiber bundle, Topology 11 (1972), 179-194. MR 45 \#4414.

2. K. Borsuk, Theory of retracts, PWN, Warsaw, 1967. MR 35 \# 7306.

3. G. E. Bredon, Introduction to compact transformation groups, Academic Press, New York, 1972.

4. T. A. Chapman, Simple homotopy-theory for ANR's (to appear).

5. J. Cheeger and J. M. Kister, Counting topological manifolds, Topology 9 (1970), 149 -151. MR 41 \# 1055.

6. M. M. Cohen, A course in simple-homotopy theory, Springer-Verlag, Berlin and New York, 1973. MR 50 \# 14762.

7. P. E. Conner and E. E. Floyd, Orbit spaces of circle groups of transformations, Ann. of Math. (2) 67 (1958), 90-98. MR 19, 1180.

8. R. C. Kirby and L. C. Siebenmann, On the triangulation of manifolds and the Hauptvermutung, Bull. Amer. Math. Soc. 75 (1969), 742-749. MR 39 \#3500.

9. L. L. Lininger, On topological transformation groups, Proc. Amer. Math. Soc. 20 (1969), 191-192. MR 38 \# 3848.

10. L. C. Siebenmann, The obstruction to finding a boundary for an open manifold of dimension greater than five, Thesis, Princeton Univ., 1965.

11. Disruption of low-dimensional handlebody theory by Rohlin's theorem, Topology of Manifolds, Markham, Chicago, Ill., 1970, pp. 57 -76. MR 42 \#6836.

12. C.T.C. Wall, Surgery on compact manifolds, Academic Press, New York, 1970.

13. J. E. West, Mapping Hilbert cube manifolds to ANR's (to appear).

Department of Mathematics, Cornell University, Ithaca, New York 14853 